\title{
The Parametric Modification of Fuzzy Sets Constituting a Content of Medical Linguistic Variables
}

\author{
Elisabeth Rakus-Andersson
}

\begin{abstract}
One of the most important features of fuzzy set theory is its potential for the modeling of natural language expressions. Most works done on this topic focus on some parts of natural language, mostly those that correspond to the socalled "evaluating linguistic expressions". We build constraints for the mathematical substitutes of these expressions to mark characteristic limits on an ordered scale.

We discuss a case of creating the total list of terms of the linguistic variable "symptom presence in diagnosis" replaced by fuzzy sets all emerging from one presence description "seldom". To accomplish this task we insert a particular parameter in the membership functions of the sets.
\end{abstract}

\section{INTRODUCTION}

In some works devoted to the creation of contents of specified lists $[1,2,7,8,9,10]$ the membership functions of fuzzy sets representing the lists' expressions are derived rather intuitively by applying symmetry for opposite atomic expressions. This method was used in the papers composed by Adlassnig [1,2], in which the diagnosis process, based on clinical symptoms, was the item of investigations. In the sequence of relation equations three fuzzy relations were involved, namely: "patient-symptom", "symptom-diagnosis" and "patient-diagnosis". The general fuzzy relation reflecting the compositional rule of inference is designed as "patient-diagnosis" = "patient-symptom" ○ "symptomdiagnosis", in which the symbol $\circ$ stands for the max-min composition of relations. First we determine contents of the relations "patient-symptom" and "symptom-diagnosis" as collections of pairs with assigned membership degrees being numbers belonging to interval $[0,1]$. Then we are capable of computing entries of relation "patient-diagnosis" that are relevant to decide of acceptance or rejection of a diagnosis considered in patient. The relation "patient-symptom" is created on the basis of clinical symptoms found in patients on condition that the lack of a symptom is marked by zero while a critical harmful extension of a biological index is followed by the value of one. It is quite easy to assign the membership degrees to the pairs ("patient", "symptom") but the development of mathematical forms of associations among the symptoms and the diagnoses is a sophisticated task to fulfill in the diagnostic model. The connection between "symptom" and "diagnosis" in each pair ("symptom", "diagnosis"), constituting a part of the relation "symptom-diagnosis", is rendered as a value of the membership degree assigned to this pair. The membership degree, in turn, expresses the relevance of "symptom" for "diagnosis". To determine intensity of the symptom

Elisabeth Rakus-Andersson is with the Blekinge Institute of Technology, School of Engineering, Department of Mathematics and Science, S-37179 Karlskrona, Sweden (phone: +46455385408; email: Elisabeth.Andersson@bth.se). influence on the diagnosis decisive character, a physician poses an essential question formulated as "How often is "symptom" found in "diagnosis"” $[1,2,6]$ ?

The physician uses his experience to answer the question by selecting verbal expressions that are included in a certain list. This contains terms of the linguistic variable "symptom presence in diagnosis" defined in a reference set $[0,100]$. Each term corresponds to the fuzzy set characterized by a particularly designed membership function.

In classical diagnostic methods all restrictions imposed on fuzzy sets are prognosticated one by one provided that a selection of parameters appearing in the membership functions maintains symmetric shapes of the functions. In one group of mentioned references [8, 9] there are indications of modifying membership functions of the expressions, e.g., generated by "often" such that "very often" or "rather often". Due to these hints we should apply the operations of concentration or dilution to the membership function of "often" to narrow or widen its shape in order to create recommended membership functions of "very often" or "often", but the supports representing the last terms still remain the same as in "often", which constitutes the disadvantage of the method. In another existing diagnostic model $[1,2]$ the constraints of fuzzy sets replacing the words from the list "symptom presence in diagnosis" are successively created by the proper selection of membership function parameters for each expression. The parameters are intuitively adjusted to warrant the regular shape of functions, and they even affect the range of the function supports.

To differ from the procedures mentioned above we intend to propose the own algorithm of a mathematical determination of constrains typical of fuzzy sets assigned to verbal descriptions of presence. First we will assume the existence of three atomic expressions "the leftmost", "in the middle" and "the rightmost" in the list [5]. After deciding the restriction of one expression we reorganize this constraint to obtain the membership functions of the other atoms.

In order to widen the list of expressions coming into existence from the atomic words we precede them with additional descriptions called hedges. To generate membership functions of sophisticated linguistic formulations, including both atomic expressions and hedges, we insert only one special parameter in the membership functions of atomic expressions. For these fuzzy sets that are expanded from atomic expressions the parameter, due to its value, causes the narrowing or widening effects in supports of the sets, and at the same time, it proportionally sculptures the set membership functions as well.

We thus derive the membership functions of atomic expressions of "symptom presence in diagnosis" in section 2. Afterwards we accomplish extensions of a number of 
constrains in Section 3. To find the contents of the matrix "symptom-diagnosis" in the form of values originating from interval $[0,1]$ we finally prepare a collection of numbers that can be set in the matrix instead of verbal descriptions of presence intensity. This item is argued in Section 4.

\section{II.MeMBERShip FunCtions OF ATOMIC EXPRESSIONS}

Let us first decide the terms of a list constructed for "symptom presence in diagnosis". We suggest "presence" = \{“never", "almost never", "very seldom", "seldom", "rather seldom", "moderately", "rather often", "often", "very often", "almost always", "always"\}. By employing the membership functions (the constraints) of fuzzy sets that correspond to the expressions collected above, we will try to find these membership degrees of the sets that represent them in the most adequate way.

Let us suggest a reference set as the range $A=\left[0, A_{l}\right]$. This contains supports of all fuzzy sets assisting the terms placed in the list "symptom presence in diagnosis". We first define three atomic expressions in $A$, i.e., "the leftmost" = "seldom", "in the middle" = "moderately" and "the rightmost" = "often". We thus propose the following constrains for "the leftmost" and "the rightmost" sets, provided that the abbreviation "se" points at the parameters of "seldom". The notion "seldom" is characterized by the membership function

$$
\begin{aligned}
& y=\mu_{\text {seldom }}(x)= \\
& \left\{\begin{array}{lll}
1 & \text { for } & x \leq \alpha_{s e}, \\
1-2\left(\frac{x-\alpha_{s e}}{\gamma_{s e}-\alpha_{s e}}\right)^{2} & \text { for } & \alpha_{s e}<x \leq \beta_{s e}, \\
2\left(\frac{x-\gamma_{s e}}{\gamma_{s e}-\alpha_{s e}}\right)^{2} & \text { for } & \beta_{s e}<x<\gamma_{s e}, \\
0 & \text { for } & x \geq \gamma_{s e},
\end{array}\right.
\end{aligned}
$$

while "often" is dependent on the parameters of "seldom" in the way proposed below [6]

$$
\begin{aligned}
& y=\mu_{\text {often" }}(x)= \\
& \begin{cases}0 & \text { for } x \leq A_{l}-\gamma_{s e} \\
2\left(\frac{x-\left(A_{l}-\gamma_{s e}\right)}{\gamma_{s e}-\alpha_{s e}}\right)^{2} & \text { for } A_{l}-\gamma_{s e}<x \leq A_{l}-\beta_{s e} \\
1-2\left(\frac{x-\left(A_{l}-\alpha_{s e}\right)}{\gamma_{s e}-\alpha_{s e}}\right)^{2} & \text { for } A_{l}-\beta_{s e}<x<A_{l}-\alpha_{s e} \\
1 & \text { for } x \geq A_{l}-\alpha_{s e}\end{cases}
\end{aligned}
$$

Eq. (1) is identical with $\mu$ 'seldom" $(x)=1-s\left(x, \alpha_{s e}, \beta_{\text {se }}, \gamma_{s e}\right)$, in which $s$ is the function of the $s$-class $[1,2,4,6,8,9]$,

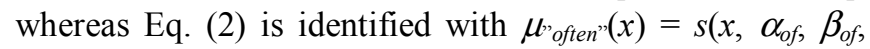
$\left.\gamma_{o f}\right)$, where $\alpha_{o f}=A_{l}-\gamma_{s e}, \beta_{o f}=A_{l}-\beta_{s e}, \gamma_{o f}=A_{l}-\alpha_{s e}$, and the abbreviation "of" is intended for the variable "often". Let us also suppose that $\gamma_{s e}=\alpha_{o f}=\frac{A_{l}}{2}$.

\section{Example 1}

We accept as a common range for all fuzzy sets the reference set $A=[0,1,2, \ldots, 100]$ as the most adaptable set to test the patients' samples. Then $A_{l}$ takes the value of 100 as the largest value in the range $A$. If we also state the values of $\alpha_{\text {se }}, \beta_{\text {se }}$ and $\gamma_{s e}$ as 30, 40 and 50, respectively, in "seldom" and "often" we then will implement the membership functions of the "leftmost" and "rightmost" atomic words derived as the split definitions

$$
\begin{aligned}
& y=\mu_{\text {seldom" }}(x)= \\
& \begin{cases}1 & \text { for } x \leq 30, \\
1-2\left(\frac{x-30}{50-30}\right)^{2} & \text { for } 30<x \leq 40, \\
2\left(\frac{x-50}{50-30}\right)^{2} & \text { for } 40<x<50, \\
0 & \text { for } x \geq 50,\end{cases}
\end{aligned}
$$

and

$$
\begin{aligned}
& y=\mu_{\text {often" }^{\prime}}(x)= \\
& \begin{cases}1 & \text { for } x \leq 100-50, \\
2\left(\frac{x-(100-50)}{50-30}\right)^{2} & \text { for } 100-50<x \leq 100-40, \\
1-2\left(\frac{x-(100-30)}{50-30}\right)^{2} & \text { for } 100-40<x<100-30, \\
0 & \text { for } x \geq 100-30 .\end{cases}
\end{aligned}
$$

We emphasize that we only need to define "the leftmost" description to generate both membership functions for "seldom" and "often".

The membership function of "moderately" is suggested to be the $\pi$-class function $[1,2,4,6,8,9]$, and is adopted here as the restriction of an atomic expression formulated as "in the middle". The mapping is affected by the parameters of "seldom" and takes a form of

$$
\begin{aligned}
& y=\mu_{\text {"moderately" }}(x)=\pi\left(x, \frac{A_{l}}{2}-\alpha_{s e}, \frac{A_{l}}{2}\right)= \\
& \left\{\begin{array}{lr}
s\left(x, \alpha_{s e}, \frac{A_{l}+2 \alpha_{s e}}{4}, \frac{A_{l}}{2}\right) & \text { for } x \leq \frac{A_{l}}{2}, \\
1-s\left(x, \frac{A_{l}}{2}, \frac{3 A_{l}-2 \alpha_{s e}}{4}, A_{l}-\alpha_{s e}\right) & \text { for } x>\frac{A_{l}}{2} .
\end{array}\right.
\end{aligned}
$$

\section{Example 2}

For $A_{l}=100$ and $\alpha_{s e}=30$, which influence the borders in (5), the last formula becomes

$$
\begin{aligned}
& y=\pi(x, 20,50)= \\
& \left\{\begin{array}{lll}
s(x, 30,40,50) & \text { for } & x \leq 50, \\
1-s(x, 50,60,70) & \text { for } & \mathrm{x}>50 .
\end{array}\right.
\end{aligned}
$$




\section{Restrictions Of Sets With Hedges In The Names}

We often need to widen the list of expressions coming into existence from the atomic words with the membership functions established in (1) and (2). If we want to use the descriptions "very seldom" or "rather seldom", then we should adjust the membership functions of new fuzzy sets that possess names consisting of both the atomic words and the hedges. The hedges are interpreted as supplementary descriptions (usually adjectives) added to atomic words. In the word compositions "very seldom" or "rather seldom", the hedges are found as "very" and "rather". To be furnished with membership functions of sophisticated linguistic formulations, including such adjectives as "very", "rather", "almost" and the like, we add a parameter $\delta$ to the parameters $\alpha_{s e}, \beta_{\text {se }}, \gamma_{\text {se }}$, already existing in (1) and (2). The action of the parameter $\delta$ causes either a narrowing or a widening effect in supports of these fuzzy sets that are derived from atomic expressions. The shapes of the membership functions defining the newly created sets are similar to genuine functions characteristic of atoms.

The rearranged membership functions of expressions belonging to a group selected as "hedge seldom" are yielded as $[5,6]$

$$
\begin{aligned}
& y=\mu_{\text {"hedge seldom" }}(x)= \\
& \begin{cases}1 & \text { for } x \leq \alpha_{s e} \delta, \\
1-2\left(\frac{x-\alpha_{s e} \delta}{\left(\gamma_{s e}-\alpha_{s e}\right) \delta}\right)^{2} & \text { for } \alpha_{s e} \delta<x \leq \beta_{s e} \delta, \\
2\left(\frac{x-\gamma_{s e} \delta}{\left(\gamma_{s e}-\alpha_{s e}\right) \delta}\right)^{2} & \text { for } \beta_{s e} \delta<x<\gamma_{s e} \delta, \\
0 & \text { for } x \geq \gamma_{s e} \delta .\end{cases}
\end{aligned}
$$

The expected constrains of the sets being members of "hedge often" are expanded in the form

$$
\begin{aligned}
& y=\mu_{\text {"hedge often" }}(x)= \\
& \begin{cases}0 & \text { for } x \leq A_{l}-\gamma_{s e} \delta, \\
2\left(\frac{x-\left(A_{l}-\gamma_{s e} \delta\right)}{\left(\gamma_{s e}-\alpha_{s e}\right) \delta}\right)^{2} & \text { for } A_{l}-\gamma_{s e} \delta<x \leq A_{l}-\beta_{s e} \delta, \\
1-2\left(\frac{x-\left(A_{l}-\alpha_{s e} \delta\right)}{\left(\gamma_{s e}-\alpha_{s e}\right) \delta}\right)^{2} & \text { for } A_{l}-\beta_{s e} \delta<x<A_{l}-\alpha_{s e} \delta, \\
1 & \text { for } x \geq A_{l}-\alpha_{s e} \delta .\end{cases}
\end{aligned}
$$

\section{Example 3}

Due to Eqs (7) and (8) we suggest the formulas of membership functions with hedges for two groups of fuzzy sets. We modify Eq. (7) as

$$
\begin{aligned}
& y=\mu_{\text {"hedge seldom" }}(x)= \\
& \begin{cases}1 & \text { for } x \leq 30 \delta \\
1-2\left(\frac{x-30 \delta}{(50-30) \delta}\right)^{2} & \text { for } 30 \delta<x \leq 40 \delta \\
2\left(\frac{x-50 \delta}{(50-30) \delta}\right)^{2} & \text { for } 40 \delta<x<50 \delta \\
0 & \text { for } x \geq 50 \delta\end{cases}
\end{aligned}
$$

to produce membership functions of the variables originating from "seldom".

The changes in (8) made as

$$
\begin{aligned}
& y=\mu_{\text {"hedge often" }}(x)= \\
& \begin{cases}0 & \text { for } x \leq 100-50 \delta, \\
2\left(\frac{x-(100-50 \delta)}{(50-30) \delta}\right)^{2} & \text { for } 100-50 \delta<x \leq 100-40 \delta, \\
1-2\left(\frac{x-(100-30 \delta)}{(50-30) \delta}\right)^{2} & \text { for } 100-40 \delta<x<100-30 \delta, \\
1 & \text { for } x \geq 100-30 \delta,\end{cases}
\end{aligned}
$$

give a class of functions possessing "often" in their names.

The parameter $\delta$ works in accordance with the following criteria $[3,5]$ :

1. $\delta=1$ where no hedge in (7) and (8) is needed (empty hedge);

2. $0<\delta<1$ is applied for hedges with narrowing effects;

3. $\delta>1$ is introduced for hedges with widening effects.

\section{Example 4}

For Eq. (9) we have tested different values of the parameter $\delta$ to finally decide that the most appropriate values of $\delta$ in the case of "seldom" can be stated as $\delta=0.75$ for "very seldom", $\delta=0.5$ for "very, very seldom" = "almost never", $\delta$ $=0.25$ for "very, very, very seldom" $=$ "never" and $\delta=1.25$ for "rather seldom". The values of $\delta<1$ will narrow supports of "hedge seldom" sets but $\delta>1$, on the contrary, enlarges an outlook of "rather seldom".

The graphs of the membership functions generated by "seldom" when taking into account the parameters designed above are depicted in Fig. 1.

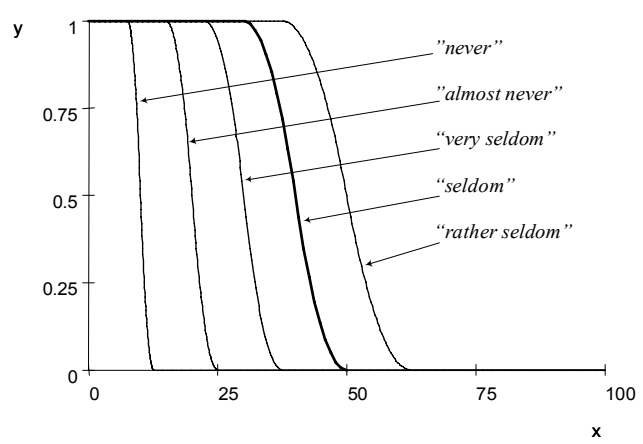

Fig. 1. The constraints of the fuzzy sets generated by "seldom" 
Formula (10) yields further composed structures proceeding from the other atomic word "often". By determining $\delta=0.75$ we find the membership function of "very often", $\delta=0.5$ gives "very, very often" = "almost always" and we get "very, very, very often" = "always" for $\delta$ $=0.25$. To create the membership function of "rather often" we exploit the widening effect of $\delta$ and adopt its value as $\delta=$ 1.25 .

The common result of employment of the parameter $\delta$ as a factor changing the membership function of "often" for the sophisticated expressions containing this word is seen in Fig. 2.

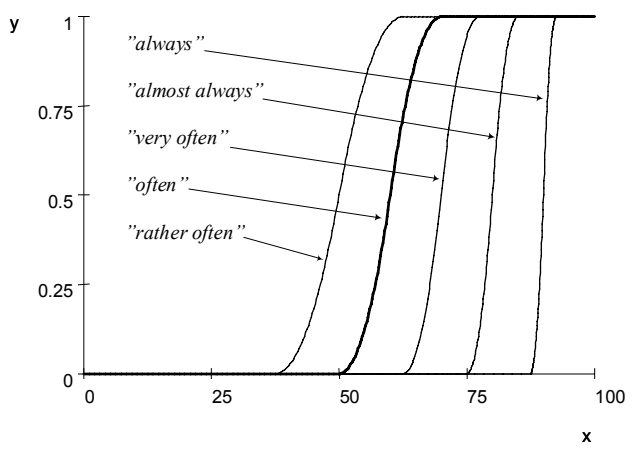

Fig. 2. The membership functions of fuzzy sets implemented by "often"

We now sample the results of all latest investigations that have led to the construction of new membership functions. These, as fuzzy sets shown in Fig. 3, represent the terms of "symptom presence in diagnosis".

\section{The Numerical Representation Of Presence INTENSITY}

In the further step of our efforts leading to a creation of the "symptom-diagnosis" matrix, we desire to extract only one value of the support that represents each fuzzy set belonging to "symptom presence in diagnosis". It seems to be reliable to accept, as a representative, this element of the set support that is assimilated to a certain border of the set's membership function. We can establish the boundary value

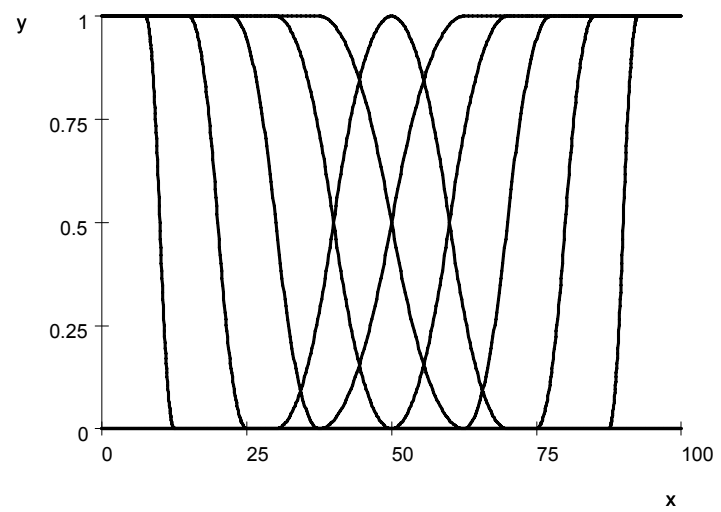

Fig. 3. The terms from the list "symptom presence in diagnosis" $x$, as the $x$-coordinate of an intersection point between the line $\mu_{s e t}(x)=1$ and a part of the membership function in which $\mu_{\text {set }}(x)<1$. The expressions coming from "seldom" have thus the borders determined as $x=\alpha_{s e} \delta=30 \delta$ while the descriptions created by "often" form the group with representatives equal to $x=A_{l}-\alpha_{s e} \delta=100-30 \delta$.

\section{Example 5}

The representatives of the sets "never", ..., "always" are sampled in Table I.

TABLE I

THE REPRESENT ATIVES OF THE SETS “NEVER", ..., "ALWAYS"

\begin{tabular}{|c|c|c|}
\hline Fuzzy sets & $\delta$ & The representatives of sets \\
\hline "never" & 0.25 & $x=30$ 「 $0.25=7.5$ \\
\hline "almost never" & 0.5 & $x=30_{\lceil\Gamma} 0.5=15$ \\
\hline "very seldom" & 0.75 & $x=30$ 『 $0.75=22.5$ \\
\hline "seldom" & 1 & $x=30\lceil 1=30$ \\
\hline "rather seldom" & 1.25 & $x=30$ 「 $1.25=37.5$ \\
\hline "moderately" & - & $x=50$ \\
\hline "rather often" & 1.25 & $x=100-30$ ॠ $1.25=62.5$ \\
\hline "often" & 1 & $x=100-30-1=70$ \\
\hline "very often" & 0.75 & $x=100-30$ \\
\hline "almost always" & 0.5 & $x=100-30$ ॠ $0.5=85$ \\
\hline "always" & 0.25 & $x=100-30$ 『 $0.25=92.5$ \\
\hline
\end{tabular}

To give expression to the verbal descriptions of presence via values of the membership degrees, we finally plan a common restriction involving the $s$-class function

$$
\mu_{\text {"common" }}(x)=s\left(x, \alpha_{\text {common }}, \beta_{\text {common }}, \gamma_{\text {common }}\right)
$$

that should completely cover the space formed for the representative values from Table 1.

\section{Example 6}

Let us accept $\alpha_{\text {common }}=7.5, \beta_{\text {common }}=50$ and $\gamma_{\text {common }}=92.5$ in (11). It should be emphasized that the borders of the space for "common" are decided to be equal to 7.5 respectively 92.5 to obtain the value of zero as the membership degree standing for "never", and the value of one assigned to "always" in accordance with the physicians' abilities to interpret "never" and "always". By setting the numbers from the last column of Table I in (11) as the $x$-values, we discover the associations between the names of variables and the corresponding membership degrees assigned to them. We state the results of appropriate computations in Table II.

TABLE II

THE NUMERICAL DESCRIPTION OF PRESENCE TERMS

\begin{tabular}{c|c|c}
\hline \hline Fuzzy sets & $x$ & $\mu$ "common" $(x)$ \\
\hline "never" & 7.5 & 0 \\
"almost never" & 15 & 0.016 \\
"very seldom" & 22.5 & 0.062 \\
"seldom" & 30 & 0.14 \\
"rather seldom" & 37.5 & 0.25 \\
"moderately" & 50 & 0.5 \\
"rather often" & 62.5 & 0.75 \\
"often" & 70 & 0.86 \\
"very often" & 77.5 & 0.938 \\
"almost always" & 85 & 0.984 \\
"always" & 92.5 & 1 \\
\hline \hline
\end{tabular}


Table II provides us with the information on how to tie the words taking place in the list "symptom presence in diagnosis", to real numbers that replace them in the fuzzy relation "symptom - diagnosis", known as "medical knowledge" of the diagnostic model.

When a physician is asked for deciding the presence of a symptom in the corresponding diagnosis, then he should only choose a word from the list containing the items that determine "symptom presence in diagnosis". In computations assisting a mathematical model one of the numbers put into the last column of Table II replaces the verbal expression approved by the physician.

To improve the comprehension of the last statements let us observe an essence of relation "symptom - diagnosis" in the next example.

\section{Example 7}

We consider three diagnoses $D_{1}=$ "high risk of cardiovascular diseases", $D_{2}=$ "coronary heart disease" and $D_{3}=$ "myocardial infarct". These are associated with important symptoms $S_{1}$ - "feeling of sickness", $S_{2}-$ "hypertension" and $S_{3}$ - "increased level of $L D L$ cholesterol".

To answer the question: "How often is $S_{j}$ found in $D_{k}$ ?", $j$, $k=1,2,3$, the physician selects a word from the list defining "symptom presence in diagnosis". His answers are collected in Table III.

TABLE III

SYMPTOM PRESENCE IN DIAGNOSIS

\begin{tabular}{c|c|c|c}
\hline \hline$S_{j} / D_{k}$ & $D_{1}$ & $D_{2}$ & $D_{3}$ \\
$S_{1}$ & almost never & very seldom & often \\
$S_{2}$ & very often & rather often & often \\
$S_{3}$ & very often & very often & almost always \\
\hline \hline
\end{tabular}

The associated relation "symptom-diagnosis" denoted by $S D$ is given as

$$
S D=S_{2} S_{1}\left[\begin{array}{ccc}
D_{1} & D_{2} & D_{3} \\
S_{3}
\end{array}\left[\begin{array}{ccc}
0.016 & 0.062 & 0.86 \\
0.938 & 0.75 & 0.86 \\
0.938 & 0.938 & 0.984
\end{array}\right] .\right.
$$

If the relation "patient-symptom" called $P S$ is found for patient $P$ as

$$
\left.\begin{array}{rcc}
S_{1} & S_{2} & S_{3} \\
P S=P[0.7 & 0.5 & 0.3
\end{array}\right]
$$

then the relation "patient-diagnosis" marked by $P D$ will be obtained as the result of compositional rule of inference $P D$ $=P S \circ S D[1,2,4,6]$ in the form of

$$
\left.\begin{array}{rcc}
S_{1} & S_{2} & S_{3} \\
P D=P[0.5 & 0.5 & 0.7
\end{array}\right] .
$$

In conformity with the rule telling us that the accepted diagnosis should possess the highest membership degree in $P D$ we accept $D_{3}$, which the physician has confirmed as well. The matrix $S D$ usually adopted as "medical knowledge" is a very important part of the diagnostic model since it constitutes the model's invariant when comparing it with different relations $P S$ constructed for individual patients.

\section{V.CONCLUSIONS}

In the composed paper we have only sketched an outline of the diagnostic process without discussing it in details since there are lots of available publications devoted to this topic. The item that has particularly appealed our attention concerns the modification of linguistic variables containing verbal expressions converted to numbers. The relation "symptom-diagnosis", which is the effect of a judgment of symptom presence in diagnosis, constitutes an essential part of medical diagnosis and should be prepared in the model with a particular care.

In the introduction we have shortly reported other methods of creating linguistic variables to begin the own model of their implementations.

At first we have shown how to find the membership functions of all atoms being of basic meaning for the list that determines the linguistic variable.

By inserting the value of the supplementary parameter $\delta$ we have constructed the membership functions of more sophisticated verbal descriptions originating from atomic words. The effect of the $\delta$-parameter insertion is essential when operating on supports of fuzzy sets. The supports become narrower or wider, which reflects our intuitive ability of perception telling us that sets possessing in the description the word "very" should be shrunk in comparison to sets deprived of this supplement.

We emphasize that only one function has been intuitively prognosticated while the other mappings have been mathematically emerged from it. This differs our method from other implementations of fuzzy sets in which patterns for generating membership functions do not exist. We thus hope that all transformations performed on the membership functions have demonstrated the logical and elegant design of the mathematical scenario, which should be regarded as an advantage of the presented model.

\section{REFERENCES}

[1] K. P. Adlassnig, "A Fuzzy Logical Model of Computer-assisted Medical Diagnosis," Methods of Information in Medicine 19, $\mathrm{nr} 3, \mathrm{pp}$. 141-148, 1980.

[2] K. P. Adlassnig, "Fuzzy Modeling and Reasoning in a Medical Diagnostic Expert System," EVD in Medizin und Biologie 17, 1/2, pp. 12-20, 1986.

[3] B. Bouchon-Meunier, "Fuzzy Logic and Knowledge Representation Using Linguistic Modifiers," in Fuzzy Logic for the Management of Uncertainty, Edited by L. A. Zadeh and J. Kacprzyk, New York: Wiley, 1992.

[4] J. Kacprzyk,. Zbiory rozmyte w analizie systemowej (Fuzzy Sets in System Analysis), Warszawa: PWN, 1986 (in Polish).

[5] V. Novák and I. Perfilieva, "Evaluating of Linguistic Expressions and Functional Fuzzy Theories in Fuzzy Logic," in Computing with Words in Information - Intelligent Systems 2, Edited by L. A. Zadeh and J. 
Kacprzyk, Studies in Fuzziness and Soft Computing Series, vol. 33, Berlin Heidelberg New York: Springer-verlag, pp. 383-406, 1999.

[6] E. Rakus-Andersson, Fuzzy and Rough Techniques in Medical Diagnosis and Medication, Berlin Heidelberg: Springer-verlag, 2007.

[7] L. A. Zadeh, "The Concept of a Linguistic Variable and its Application to Approximate Reasoning," Inf. Sci. 8, pp. 199-249, 1975.

[8] L. A. Zadeh, Calculus of Fuzzy Restrictions, Fuzzy Sets and Their Applications to Cognitive and Decision Processes, London: Academic Press, 1975.

[9] L. A. Zadeh, "A Computational Approach to Fuzzy Quantifiers in Natural Languages," Computers and Mathematics 9, pp. 149-184, 1983.

[10] L. A. Zadeh, "Test-score Semantics as a Basis for a Computational Approach to the Representation of Meaning," Literary and Linguistic Computing 1, pp. 24-35, 1986.

[11] L. A. Zadeh, "From Computing with Numbers to Computing with Words - From Manipulation of Measurements to Manipulation of Perceptions," IEEE Transactions on Circuits and Systems 45, pp. 105119, 1999.

[12] H. J. Zimmermann, Fuzzy Set Theory and Its Applications, $3^{\text {rd }}$ edn, Boston: Kluwer Academic Publishers, 1996. 